\title{
Facile Synthesis, Antioxidant and Antimicrobial Activity of Amino Methylene Bisphosphonates
}

\author{
Uma Maheswara Rao Kunda, ${ }^{a}$ Satheesh Krishna Balam, ${ }^{a}$ Bakthavatchala Reddy Nemallapudi, ${ }^{a}$ \\ Syama Sundar Chereddy, ${ }^{a}$ Sandip Kumar Nayak, ${ }^{b}$ and Suresh Reddy Cirandur*,a \\ ${ }^{a}$ Department of Chemistry, Sri Venkateswara University; Tirupati 517 502, India: and ${ }^{b}$ Bio-Organic Division, BARC; \\ Mumbai 400-085, India. Received September 8, 2011; accepted October 17, 2011; published online October 21, 2011
}

\begin{abstract}
A green and efficient preparation method for the amino bisphosphonates is accomplished by simple mixing and stirring of diethylphosphite, triethylorthoformate and various amines in the presence of amberlyst-15 as catalyst at room temperature under solvent free conditions. The title compounds are characterized by IR, ${ }^{1} \mathrm{H}-,{ }^{13} \mathrm{C}-,{ }^{31} \mathrm{P}-\mathrm{NMR}$ and mass spectra, also studied their antimicrobial and antioxidant activity.
\end{abstract}

Key words amino methylene bisphosphonate; antimicrobial activity; antioxidant activity; amberlyst-15

Early research on the role of antioxidants in biology focused on their use in preventing oxidation of unsaturated fats, which is the cause of rancidity. ${ }^{1)}$ However, it was the identification of the antioxidant activity of vitamins $\mathrm{A}, \mathrm{C}$, and $\mathrm{E}$ that revolutionized the field and led to the realization of the importance of antioxidants in the biochemistry of living organisms. ${ }^{2,3)}$ The antioxidant activity was measured simply by placing the fat in a closed container with oxygen and measuring the rate of oxygen consumption. Excessive generation of reactive oxygen species (ROS) induced by various stimuli leads to variety of pathophysiological abnormalities such as inflammation, diabetes, genotoxicity and cancer. Search for active components that prevent or reduce the impact of oxidative stress on cell is a contemporary field.

The aim of this study was to investigate the in vitro antimicrobial and antioxidant profile of different nitrogen-containing bisphosphonates (N-BPS), which, the best of our knowledge has not yet been systematically studied. N-BPS, a class of geminal bisphosphonates (BPS) are important drugs for wide variety of medicinal applications. ${ }^{4)}$ Besides their well-known anti-bone resorption properties, ${ }^{5)}$ antimicrobial activity ${ }^{6}$ and anti-tumor properties, ${ }^{7)}$ some of their derivatives inhibit cancer manifestations through antiangiogenic, anti-invasive and immunomodulatory actions. ${ }^{8-10)}$

BPS are also effective in the treatment of rheumatoid arthritis (RA). ${ }^{11-13)}$ Recently bisphosphonates have been proved to prevent glucocorticoid-induced osteoporosis in RA ${ }^{14,15)}$ and to possess selective antioxidant properties in vitro. ${ }^{16)}$ Moreover, some recent clinical studies in the animals indicate antiinflammatory effects by BPS in RA. ${ }^{16,17)}$

In recent years, use of solid acid catalysts has attracted considerable attention. ${ }^{18)}$ In this regard, amberlyst- 15 possesses unique properties is successfully used as a solid acid catalyst for the preparation of bisphosphonates from the amine, dialkyl phosphite and triethylorthoformate. Amberlyst-15 is a strongly acidic, sulfonic acid, macro reticular polymeric resin based on cross linked styrene divinylbenzene copolymers. Its continuous open pore structure and excellent physical, thermal and chemical stability makes it the resin of choice in many applications. It also possesses greater resistance to oxidants such as chlorine, oxygen and chromates than most other polymeric resins. Its physical and chemical stability, non corrosive, non toxic nature, selectivity in reactions, reusability and environmental compatibility make this as a versatile catalyst for per- forming the various functional group transformations in the general organic synthesis. ${ }^{19)}$

We now report a relatively simple synthesis of amino methylene bisphosphonates in good yields using amberlyst-15 as an efficient and environmentally benign catalyst under solventfree conditions at room temperature. There have been no reports on the synthesis of amino methylene bisphosphonates using amberlyst-15 as catalyst.

\section{Experimental}

General Melting points were recorded on Buchi R-535 apparatus and are uncorrected. IR spectra were recorded on a Perkin-Elmer FT-IR 240-c spectrophotometer using $\mathrm{KBr}$ optics. ${ }^{1} \mathrm{H}-,{ }^{13} \mathrm{C}$ - and ${ }^{31} \mathrm{P}-\mathrm{NMR}$ spectra were recorded on AMX $400 \mathrm{MHz}$ NMR spectrometers operating at $400 \mathrm{MHz}$ for ${ }^{1} \mathrm{H}-$, $100 \mathrm{MHz}$ for ${ }^{13} \mathrm{C}$ - and $161.89 \mathrm{MHz}$ for ${ }^{31} \mathrm{P}-\mathrm{NMR}$. NMR data were recorded in DMSO- $d_{6}$ and referenced to tetramethylsilane (TMS) $\left({ }^{1} \mathrm{H}\right.$ and $\left.{ }^{13} \mathrm{C}\right)$ and $85 \% \mathrm{H}_{3} \mathrm{PO}_{4}\left({ }^{31} \mathrm{P}\right)$. Mass spectra were recorded on a Finnigan MAT 1020/Micro-Mass Q-T of micro AMPS MAX 10/6A, Hz 60/50 system fitted with a built-in inlet system. Elemental analyses were performed using Perkin Elmer 2400 instrument at the Central Drug Research Institute (CDRI), Lucknow, India.

Experimental Procedure for the Preparation of Tetraethyl(3,4-dichlorophenylamino)methylenebisphosphonate (3a) A mixture of 3,4-dichloro aniline (1a, $0.005 \mathrm{~mol})$, diethylphosphite $(2,0.01 \mathrm{~mol})$, triethylorthoformate $(0.005 \mathrm{~mol})$ and catalytic amount of amberlyst-15 (0.1 g) was stirred magnetically at room temperature for appropriate time. After completion of the reaction as monitored by TLC, it was purified by column chromatography on $60-120$ mesh silica gel using ethyl acetate-hexane $(1: 4)$ as eluent. The residue was recrystallized from ethylacetate to afford pure $\mathbf{3 a}$ in $95 \%$ yield. This procedure was applied successfully for the preparation of $\mathbf{3 b}-\mathbf{j}$. All the compounds were characterized by IR, ${ }^{1} \mathrm{H}-,{ }^{13} \mathrm{C}-$, ${ }^{31} \mathrm{P}-\mathrm{NMR}$, mass spectral and elemental analytical data.

Tetraethyl(3,4-dichlorophenylamino)methylenebisphosphonate (3a): White solid, yield: $95 \%, \mathrm{mp} 120-122^{\circ} \mathrm{C} .{ }^{1} \mathrm{H}-$ NMR $\left(400 \mathrm{MHz}, \mathrm{DMSO}-d_{6}\right) \delta: 1.06\left(6 \mathrm{H}, \mathrm{t},{ }^{3} J_{\mathrm{PH}}=8.8 \mathrm{~Hz}\right.$, $\left.\mathrm{POCH}_{2} \mathrm{CH}_{3}\right), 1.20\left(6 \mathrm{H}, \mathrm{t},{ }^{3} J_{\mathrm{PH}}=9.2 \mathrm{~Hz}, \mathrm{POCH}_{2} \underline{\mathrm{CH}}_{3}\right), 3.70-$ $3.89\left(8 \mathrm{H}, \mathrm{m}, \mathrm{P}-\mathrm{OCH}_{2} \mathrm{CH}_{3}\right), 4.80-5.04(1 \mathrm{H}, \mathrm{m}, \mathrm{PCH}), 5.90$ $(1 \mathrm{H}, \mathrm{s}, \mathrm{NH}), 6.25-6.55(3 \mathrm{H}, \mathrm{m}, \mathrm{Ar}-\mathrm{H}) .{ }^{13} \mathrm{C}-\mathrm{NMR}(100 \mathrm{MHz}$, DMSO- $\left.d_{6}\right) \delta$ : $14.8\left(\mathrm{~d},{ }^{3} J_{\mathrm{P}-\mathrm{C}}=5.8 \mathrm{~Hz}, \mathrm{P}-\mathrm{OCH}_{2}-\mathrm{CH}_{3}\right), 57.2(\mathrm{t}$, $\left.{ }^{1} J_{\mathrm{PC}}=156.3 \mathrm{~Hz}, \mathrm{PCH}\right), 59.5\left(\mathrm{~d},{ }^{2} J_{\mathrm{P}-\mathrm{C}}=7.6 \mathrm{~Hz}, \mathrm{P}-\mathrm{OCH}_{2} \mathrm{CH}_{3}\right)$, 
112.1 (C-6), 111.9 (C-5), 116.4 (C-2), 122.2 (C-4), 125.6 (C-3), $138.5(\mathrm{C}-1) .{ }^{31} \mathrm{P}-\mathrm{NMR}\left(161.7 \mathrm{MHz}, \mathrm{DMSO}-d_{6}\right) \delta$ : 18.26. IR $(\mathrm{KBr}) \mathrm{cm}^{-1}: 3200(\mathrm{NH}), 1240(\mathrm{P}=\mathrm{O}), 760\left(\mathrm{P}-\mathrm{C}_{\text {aliphatic }}\right)$. Electrospray ionization (ESI)-MS $(\mathrm{m} / \mathrm{z}): 447\left(\mathrm{M}^{+}\right)$. Anal. Calcd for $\mathrm{C}_{15} \mathrm{H}_{25} \mathrm{Cl}_{2} \mathrm{NO}_{6} \mathrm{P}_{2}$ : C, 40.20; $\mathrm{H}, 5.62 ; \mathrm{N}, 3.12$. Found $\mathrm{C}$, 40.16; $\mathrm{H}, 5.58 ; \mathrm{N}, 3.08$.

Tetraethyl(1-phenylethylamino)methylenebis-phosphonate (3b): White solid, yield: $70 \%$, mp $135-137^{\circ} \mathrm{C} .{ }^{1} \mathrm{H}-\mathrm{NMR}$ $\left(400 \mathrm{MHz}, \mathrm{DMSO}-d_{6}\right) \delta: 1.15\left(6 \mathrm{H}, \mathrm{t},{ }^{3} \mathrm{~J}_{\mathrm{PH}}=9.1 \mathrm{~Hz}, \mathrm{POCH}_{2} \mathrm{CH}_{3}\right)$, $1.22\left(3 \mathrm{H}, \quad \mathrm{d}, \quad{ }^{2} J_{\mathrm{H}-\mathrm{H}}=7.4 \mathrm{~Hz}\right), \quad 1.25\left(6 \mathrm{H}, \quad \mathrm{t},{ }^{3} J_{\mathrm{PH}}=9.2 \mathrm{~Hz}\right.$, $\left.\mathrm{POCH}_{2} \mathrm{CH}_{3}\right), 1.78\left(1 \mathrm{H}, \mathrm{q}, J=7.6 \mathrm{~Hz}, \mathrm{Ph}-\underline{\mathrm{CH}}-\mathrm{CH}_{3}\right), 3.82-4.05$ $\left(8 \mathrm{H}, \mathrm{m}, \mathrm{P}-\mathrm{OCH}_{2} \mathrm{CH}_{3}\right), 4.80-5.05(1 \mathrm{H}, \mathrm{m}, \mathrm{PCH}), 5.65(1 \mathrm{H}, \mathrm{s}$, $\mathrm{NH}), 6.25-7.15$ (5H, m, Ar-H). ${ }^{13} \mathrm{C}-\mathrm{NMR}(100 \mathrm{MHz}, \mathrm{DMSO}-$ $\left.d_{6}\right) \delta: 13.7,16.2\left(\mathrm{~d},{ }^{3} J_{\mathrm{P}-\mathrm{C}}=5.8 \mathrm{~Hz}, \mathrm{P}-\mathrm{OCH}_{2}-\mathrm{CH}_{3}\right), 45.1,58.3$ $\left(\mathrm{t},{ }^{1} J_{\mathrm{PC}}=157.8 \mathrm{~Hz}, \mathrm{PCH}\right), 61.2\left(\mathrm{~d},{ }^{2} J_{\mathrm{P}-\mathrm{C}}=7.6 \mathrm{~Hz}, \mathrm{P}-\mathrm{OCH}_{2} \mathrm{CH}_{3}\right)$, 114.4 (C-4), 114.7 (C-2), 115.2 (C-3), 115.9 (C-5), 116.2 (C-6), $136.8(\mathrm{C}-1) .{ }^{31} \mathrm{P}-\mathrm{NMR}\left(161.7 \mathrm{MHz}, \mathrm{DMSO}-d_{6}\right) \delta$ : 18.50. IR $(\mathrm{KBr}) \mathrm{cm}^{-1}: 3200(\mathrm{NH}), 1225(\mathrm{P}=\mathrm{O}), 775\left(\mathrm{P}-\mathrm{C}_{\text {aliphatic }}\right)$. ESIMS $(m / z)$ : $407\left(\mathrm{M}^{+}\right)$. Anal. Calcd for $\mathrm{C}_{17} \mathrm{H}_{28} \mathrm{~N}_{4} \mathrm{O}_{6} \mathrm{P}_{2}:$ C, 50.12; $\mathrm{H}, 7.67$; N, 3.44. Found C, 50.08; H, 7.63; N, 3.39.

Tetraethyl(3,4-dimethylphenylamino)methylenebisphosphonate (3c): White solid, yield: $75 \%$, mp $140-142^{\circ} \mathrm{C}$. ${ }^{1} \mathrm{H}-\mathrm{NMR}$ $\left(400 \mathrm{MHz}, \mathrm{DMSO}-d_{6}\right) \delta: 1.13\left(6 \mathrm{H}, \mathrm{t},{ }^{3} \mathrm{~J}_{\mathrm{PH}}=9.0 \mathrm{~Hz}, \mathrm{POCH}_{2} \mathrm{CH}_{3}\right)$, $1.22(3 \mathrm{H}, \mathrm{s}), \quad 1.24(3 \mathrm{H}, \mathrm{s}), 1.26\left(6 \mathrm{H}, \mathrm{t},{ }^{3} J_{\mathrm{PH}}=9.2 \mathrm{~Hz}\right.$, $\left.\mathrm{POCH}_{2} \mathrm{CH}_{3}\right), 3.70-3.99\left(8 \mathrm{H}, \mathrm{m}, \mathrm{P}-\mathrm{OCH}_{2} \mathrm{CH}_{3}\right), 4.75-4.99$ $(1 \mathrm{H}, \mathrm{m}, \mathrm{PCH}), 5.55$ (1H, s, NH), 6.78-7.30 (4H, m, Ar-H). ${ }^{13} \mathrm{C}-\mathrm{NMR} \quad\left(100 \mathrm{MHz}, \mathrm{DMSO}-d_{6}\right) \delta: 16.5\left(\mathrm{~d},{ }^{3} J_{\mathrm{P}-\mathrm{C}}=5.8 \mathrm{~Hz}\right.$, $\left.\mathrm{P}-\mathrm{OCH}_{2}-\mathrm{CH}_{3}\right), 23.5,26.4,56.0 \quad\left(\mathrm{t},{ }^{1} J_{\mathrm{PC}}=163.6 \mathrm{~Hz}, \mathrm{PCH}\right)$, $63.0\left(\mathrm{~d},{ }^{2} \bar{J}_{\mathrm{P}-\mathrm{C}}=7.6 \mathrm{~Hz}, \mathrm{P}-\mathrm{OCH}_{2} \mathrm{CH}_{3}\right), 109.0(\mathrm{C}-2), 117.0(\mathrm{C}-5)$, 123.2 (C-6), 136.5 (C-3), 144.2 (C-4), 145.2 (C-1),. ${ }^{31} \mathrm{P}-\mathrm{NMR}$ $\left(161.7 \mathrm{MHz}, \mathrm{DMSO}-d_{6}\right) \delta: 20.52$. IR $(\mathrm{KBr}) \mathrm{cm}^{-1}: 3250(\mathrm{NH})$, $1230(\mathrm{P}=\mathrm{O}), 775\left(\mathrm{P}-\mathrm{C}_{\text {aliphatic }}\right)$. ESI-MS $(\mathrm{m} / \mathrm{z}): 407\left(\mathrm{M}^{+}\right)$. Anal. Calcd for $\mathrm{C}_{17} \mathrm{H}_{31} \mathrm{NO}_{6} \mathrm{P}_{2}: \mathrm{C}, 50.12 ; \mathrm{H}, 7.67 ; \mathrm{N}, 3.44$. Found $\mathrm{C}$, 50.08; H, 7.62; N, 3.41.

Tetraethyl(2-methyloxazol-5-yllamino)methylenebisphosphonate (3d): White solid, yield: $72 \%$, mp $160-162^{\circ} \mathrm{C}$. ${ }^{1} \mathrm{H}-\mathrm{NMR}\left(400 \mathrm{MHz}, \mathrm{DMSO}-d_{6}\right) \delta: 1.10\left(6 \mathrm{H}, \mathrm{t},{ }^{3} J_{\mathrm{PH}}=9.1 \mathrm{~Hz}\right.$, $\left.\mathrm{POCH}_{2} \mathrm{CH}_{3}\right), \quad 1.20 \quad\left(6 \mathrm{H}, \quad \mathrm{t}, \quad{ }^{3} J_{\mathrm{PH}}=9.2 \mathrm{~Hz}, \quad \mathrm{P}-\mathrm{OCH}_{2} \mathrm{C}_{3}\right)$, $1.34(3 \mathrm{H}, \mathrm{s}), 3.72-3.95\left(8 \mathrm{H}, \mathrm{m}, \mathrm{P}-\mathrm{OCH}_{2} \mathrm{CH}_{3}\right), 4.90-5.25$ $(1 \mathrm{H}, \mathrm{m}, \mathrm{PCH}), 5.50(1 \mathrm{H}, \mathrm{s}, \mathrm{NH}), 8.25\left(1 \mathrm{H}, \mathrm{s}\right.$ Ar-H). ${ }^{13} \mathrm{C}-$ NMR $\left(100 \mathrm{MHz}, \mathrm{DMSO}-d_{6}\right) \delta: 17.05\left(\mathrm{~d},{ }^{3} J_{\mathrm{P}-\mathrm{C}}=5.6 \mathrm{~Hz}, \mathrm{P}-\right.$ $\left.\mathrm{OCH}_{2}-\mathrm{CH}_{3}\right), 28.7,55.0\left(\mathrm{t},{ }^{1} J_{\mathrm{PC}}=163.4 \mathrm{~Hz}, \mathrm{PCH}\right), 62.2(\mathrm{~d}$, $\left.{ }^{2} J_{\mathrm{P}-\mathrm{C}}=7.4 \mathrm{~Hz}, \mathrm{P}-\mathrm{OCCH}_{2} \mathrm{CH}_{3}\right), 135.5$ (C-5), 147.5 (C-4), 163.0 (C-2). ${ }^{31} \mathrm{P}-\mathrm{NMR}\left(161.7 \mathrm{MHz}, \mathrm{DMSO}-d_{6}\right) \delta: 19.52$. IR $(\mathrm{KBr})$ $\mathrm{cm}^{-1}$ : $3255(\mathrm{NH}), 1235(\mathrm{P}=\mathrm{O}), 770\left(\mathrm{P}-\mathrm{C}_{\text {aliphatic }}\right)$. ESI-MS $(\mathrm{m} / \mathrm{z})$ : $384\left(\mathrm{M}^{+*}\right)$. Anal. Calcd for $\mathrm{C}_{13} \mathrm{H}_{26} \mathrm{~N}_{2} \mathrm{O}_{7} \mathrm{P}_{2}: \mathrm{C}, 40.63 ; \mathrm{H}, 6.82 ; \mathrm{N}$, 7.29. Found C, 40.59; H, 6.78; N, 7.24.

Tetraethyl(4-nitrophenylamino)methylenebisphosphonate (3e): Yellow solid, yield: $70 \%, \mathrm{mp} 205-206^{\circ} \mathrm{C} .{ }^{1} \mathrm{H}-$ NMR $\left(400 \mathrm{MHz}, \mathrm{DMSO}-d_{6}\right) \delta: 1.12\left(6 \mathrm{H}, \mathrm{t},{ }^{3} J_{\mathrm{PH}}=8.7 \mathrm{~Hz}\right.$, $\left.\mathrm{POCH}_{2} \mathrm{CH}_{3}\right), 1.22\left(6 \mathrm{H}, \mathrm{t},{ }^{3} J_{\mathrm{PH}}=9.2 \mathrm{~Hz}, \mathrm{POCH}_{2} \mathrm{CH}_{3}\right), 3.85-$ $4.10\left(8 \mathrm{H}, \mathrm{m}, \mathrm{P}-\mathrm{OC} \underline{\mathrm{H}}_{2} \mathrm{CH}_{3}\right), 4.98(1 \mathrm{H}, \mathrm{s}, \mathrm{NH}), 5.10-5.55(1 \mathrm{H}$, $\mathrm{m}, \mathrm{PCH}), 6.52-7.13$ (4H, m, Ar-H). ${ }^{13} \mathrm{C}-\mathrm{NMR}(100 \mathrm{MHz}$, $\left.\mathrm{DMSO}-d_{6}\right) \delta: 16.5\left(\mathrm{~d},{ }^{3} J_{\mathrm{P}-\mathrm{C}}=5.6 \mathrm{~Hz}, \mathrm{P}-\mathrm{OCH}_{2}-\mathrm{CH}_{3}\right), 47.8(\mathrm{t}$, $\left.{ }^{1} J_{\mathrm{PC}}=161.5 \mathrm{~Hz}, \mathrm{PCH}\right), 62.7\left(\mathrm{~d},{ }^{2} J_{\mathrm{P}-\mathrm{C}}=7.6 \mathrm{~Hz}, \mathrm{P}-\mathrm{OCH}_{2} \mathrm{CH}_{3}\right)$, 112.8 (C-2), 113.1 (C-6), 126.6 (C-5), 127.4 (C-3), 134.2 (C-4), $151.2(\mathrm{C}-1) .{ }^{31} \mathrm{P}-\mathrm{NMR}\left(161.7 \mathrm{MHz}, \mathrm{DMSO}-d_{6}\right) \delta$ : 21.5. IR $(\mathrm{KBr})$ $\mathrm{cm}^{-1}$ : $3255(\mathrm{NH}), 1230(\mathrm{P}=\mathrm{O}), 765\left(\mathrm{P}-\mathrm{C}_{\text {aliphatic }}\right)$. ESI-MS m/z: $424\left(\mathrm{M}^{+*}\right)$. Anal. Calcd for $\mathrm{C}_{15} \mathrm{H}_{26} \mathrm{~N}_{2} \mathrm{O}_{8} \mathrm{P}_{2}: \mathrm{C}, 42.46 ; \mathrm{H}, 6.18 ; \mathrm{N}$, 6.60. Found C, 42.43; H, 6.14; N, 6.56 .

Tetraethyl(4-fluorophenylamino)methylenebisphosphonate (3f): White solid, yield: $80 \%$, mp $60-61{ }^{\circ} \mathrm{C} .{ }^{1} \mathrm{H}-\mathrm{NMR}$ $\left(400 \mathrm{MHz}, \mathrm{DMSO}-d_{6}\right) \delta: 1.08\left(6 \mathrm{H}, \mathrm{t},{ }^{3} J_{\mathrm{PH}}=8.2 \mathrm{~Hz}, \mathrm{POCH}_{2} \mathrm{CH}_{3}\right)$, $1.28\left(6 \mathrm{H}, \mathrm{t},{ }^{3} J_{\mathrm{PH}}=9.2 \mathrm{~Hz}, \mathrm{POCH}_{2} \mathrm{CH}_{3}\right), 3.60-4.10(8 \mathrm{H}, \mathrm{m}$, $\left.\mathrm{P}-\mathrm{OCH}_{2} \mathrm{CH}_{3}\right), 4.85-5.08(1 \mathrm{H}, \mathrm{m}, \mathrm{PCH}), 5.95(1 \mathrm{H}, \mathrm{s}, \mathrm{NH})$, $7.48-8.20(4 \mathrm{H}, \mathrm{m}, \mathrm{Ar}-\mathrm{H}) .{ }^{13} \mathrm{C}-\mathrm{NMR}\left(100 \mathrm{MHz}, \mathrm{DMSO}-d_{6}\right) \delta$ : $17.5\left(\mathrm{~d},{ }^{3} J_{\mathrm{P}-\mathrm{C}}=5.6 \mathrm{~Hz}, \mathrm{P}-\mathrm{OCH}_{2}-\underline{\mathrm{CH}}_{3}\right), 56.7\left(\mathrm{t},{ }^{1} J_{\mathrm{PC}}=162.4 \mathrm{~Hz}\right.$, $\mathrm{PCH}), 63.2\left(\mathrm{~d},{ }^{2} J_{\mathrm{P}-\mathrm{C}}=7.6 \mathrm{~Hz}, \quad \mathrm{P}-\mathrm{OCH}_{2} \mathrm{CH}_{3}\right), 114.5(\mathrm{C}-4)$, 120.0 (C-6), 121.5 (C-5), 157.6 (C-3), 175.6 (C-1). ${ }^{31} \mathrm{P}-\mathrm{NMR}$ $\left(161.7 \mathrm{MHz}, \mathrm{DMSO}-d_{6}\right) \delta$ : 24.5; IR $(\mathrm{KBr}) \mathrm{cm}^{-1}: 3390(\mathrm{NH})$, $1244(\mathrm{P}=\mathrm{O}), 746\left(\mathrm{P}-\mathrm{C}_{\text {aliphatic }}\right)$. ESI-MS m/z: $397\left(\mathrm{M}^{+*}\right)$. Anal. Calcd for $\mathrm{C}_{15} \mathrm{H}_{26} \mathrm{FNO}_{6} \mathrm{P}_{2}: \mathrm{C}, 45.34 ; \mathrm{H}, 6.60 ; \mathrm{N}, 3.53$. Found $\mathrm{C}$, 45.29; H, 6.56; N, 3.50.

Tetraethyl(benzo[d]thiazol-2-ylamino)methylenebisphosphonate (3g): White solid, yield: $77 \%, \mathrm{mp} 125-127^{\circ} \mathrm{C}$. ${ }^{1} \mathrm{H}-\mathrm{NMR}\left(400 \mathrm{MHz}, \mathrm{DMSO}-d_{6}\right) \delta: 1.09\left(6 \mathrm{H}, \mathrm{t},{ }^{3} \mathrm{~J}_{\mathrm{PH}}=8.8 \mathrm{~Hz}\right.$, $\left.\mathrm{POCH}_{2} \mathrm{CH}_{3}\right), 1.20\left(6 \mathrm{H}, \mathrm{t},{ }^{3} J_{\mathrm{PH}}=9.2 \mathrm{~Hz}, \mathrm{POCH}_{2} \underline{\mathrm{CH}}_{3}\right), 3.75-$ $3.99\left(8 \mathrm{H}, \mathrm{m}, \mathrm{P}-\mathrm{OCH}_{2} \mathrm{CH}_{3}\right), 4.70-4.95(1 \mathrm{H}, \mathrm{m}, \mathrm{PCH}), 5.60$ $(1 \mathrm{H}, \mathrm{s}, \mathrm{NH}), 7.85-8.15(4 \mathrm{H}, \mathrm{m}, \mathrm{Ar}-\mathrm{H}) .{ }^{13} \mathrm{C}-\mathrm{NMR}(100 \mathrm{MHz}$, DMSO- $\left.d_{6}\right) \delta: 16.9\left(\mathrm{~d},{ }^{3} J_{\mathrm{P}-\mathrm{C}}=5.6 \mathrm{~Hz}, \mathrm{P}-\mathrm{OCH}_{2}-\mathrm{CH}_{3}\right), 54.4(\mathrm{~d}$, $\left.{ }^{1} J_{\mathrm{PC}}=162.4 \mathrm{~Hz}, \mathrm{PCH}\right), 65.1\left(\mathrm{~d},{ }^{2} J_{\mathrm{P}-\mathrm{C}}=7.6 \mathrm{~Hz}, \mathrm{P}-\mathrm{O}_{-} \mathrm{H}_{2} \mathrm{CH}_{3}\right)$, 113.8 (C-4), 117.6 (C-7), 121.6 (C-6), 122.1 (C-5), 131.6 (C-8), 156.7 (C-3), $174.5(\mathrm{C}-1) .{ }^{31} \mathrm{P}-\mathrm{NMR}\left(161.7 \mathrm{MHz}, \mathrm{DMSO}-d_{6}\right) \delta$ : 20.5. IR (KBr) cm $\mathrm{cm}^{-1}: 3350(\mathrm{NH}), 1235(\mathrm{P}=\mathrm{O}), 755\left(\mathrm{P}-\mathrm{C}_{\text {aliphatic }}\right)$. ESI-MS m/z: $436\left(\mathrm{M}^{+}\right)$. Anal. Calcd for $\mathrm{C}_{16} \mathrm{H}_{26} \mathrm{~N}_{2} \mathrm{O}_{6} \mathrm{P}_{2} \mathrm{~S}: \mathrm{C}$, 44.04; H, 6.01; N, 6.42. Found C, 44.01; H, 5.96; N, 6.38.

Tetraethyl(1,5-dimethyl-3-oxo-2-phenylpyrazolidin-4-ylamino)methylenebisphosphonate (3h): White solid, yield: $70 \%$, mp $139-141^{\circ} \mathrm{C}$. ${ }^{1} \mathrm{H}-\mathrm{NMR}\left(400 \mathrm{MHz}, \mathrm{DMSO}-d_{6}\right) \delta: 1.05$ $\left(6 \mathrm{H}, \mathrm{t},{ }^{3} \mathrm{~J}_{\mathrm{PH}}=9.2 \mathrm{~Hz}, \mathrm{POCH}_{2} \mathrm{CH}_{3}\right), 1.20\left(6 \mathrm{H}, \mathrm{t},{ }^{3} J_{\mathrm{PH}}=9.2 \mathrm{~Hz}\right.$, $\left.\mathrm{POCH}_{2} \mathrm{CH}_{3}\right), 1.48(3 \mathrm{H}, \mathrm{d}, J=7.5 \mathrm{~Hz}), 1.64-1.86(1 \mathrm{H}, \mathrm{m})$, $1.92-2.15(1 \mathrm{H}, \mathrm{m}), 2.52\left(3 \mathrm{H}, \mathrm{s}, \mathrm{N}-\mathrm{CH}_{3}\right), 3.70-3.92(8 \mathrm{H}$, $\left.\mathrm{m}, \mathrm{P}-\mathrm{OCH}_{2} \mathrm{CH}_{3}\right), 4.85-5.10(1 \mathrm{H}, \mathrm{m}, \mathrm{PCH}), 5.29(1 \mathrm{H}, \mathrm{s}, \mathrm{NH})$, $6.86-7.91(5 \mathrm{H}, \mathrm{m}, \mathrm{Ar}-\mathrm{H}) .{ }^{13} \mathrm{C}-\mathrm{NMR}\left(100 \mathrm{MHz}, \mathrm{DMSO}-d_{6}\right)$ $\delta: 11.6\left(\mathrm{CH}_{3}\right), 14.3\left(\mathrm{~d},{ }^{3} J_{\mathrm{P}-\mathrm{C}}=5.6 \mathrm{~Hz}, \mathrm{P}-\mathrm{OCH}_{2}-\mathrm{CH}_{3}\right), 38.4$ $\left(\mathrm{N}-\underline{\mathrm{CH}}_{3}\right), 44.6(\mathrm{C}-2), 56.4\left(\mathrm{t},{ }^{1} J_{\mathrm{PC}}=156.7 \mathrm{~Hz}, \mathrm{PCH}\right), 58.7(\mathrm{C}-1)$, $60.3\left(\mathrm{~d},{ }^{2} J_{\mathrm{P}-\mathrm{C}}=7.6 \mathrm{~Hz}, \mathrm{P}-\mathrm{OCH}_{2} \mathrm{CH}_{3}\right), 114.5\left(\mathrm{C}-3^{\prime}\right), 114.9\left(\mathrm{C}-5^{\prime}\right)$, $116.8\left(\mathrm{C}-4^{\prime}\right), 119.8\left(\mathrm{C}-2^{\prime}\right), 120.2\left(\mathrm{C}-6^{\prime}\right), 122.1\left(\mathrm{C}-1^{\prime}\right), 152.3(\mathrm{C}-$ 5). ${ }^{31} \mathrm{P}-\mathrm{NMR}\left(161.7 \mathrm{MHz}, \mathrm{DMSO}-d_{6}\right) \delta: 20.8$. IR $(\mathrm{KBr}) \mathrm{cm}^{-1}$ : $3430(\mathrm{NH}), 1220(\mathrm{P}=\mathrm{O}), 755\left(\mathrm{P}-\mathrm{C}_{\text {aliphatic }}\right)$. ESI-MS $(\mathrm{m} / \mathrm{z}): 491$ $\left(\mathrm{M}^{+}\right)$. Anal. Calcd for $\mathrm{C}_{20} \mathrm{H}_{35} \mathrm{~N}_{3} \mathrm{O}_{7} \mathrm{P}_{2}: \mathrm{C}, 48.88 ; \mathrm{H}, 7.18 ; \mathrm{N}$, 8.55. Found C, 48.84; H, 7.14; N, 8.51.

Tetraethyl(3,5-dichloro-4-hydroxyphenylamino)methylenebisphosphonate (3i): White solid, yield: $80 \%, \mathrm{mp} 114-$ $116^{\circ} \mathrm{C}$. ${ }^{1} \mathrm{H}-\mathrm{NMR}\left(400 \mathrm{MHz}, \mathrm{DMSO}-d_{6}\right) \delta: 1.09\left(6 \mathrm{H}, \mathrm{t},{ }^{3} J_{\mathrm{PH}}=\right.$ $\left.8.8 \mathrm{~Hz}, \mathrm{POCH}_{2} \mathrm{CH}_{3}\right), 1.20\left(6 \mathrm{H}, \mathrm{t},{ }^{3} J_{\mathrm{PH}}=9.2 \mathrm{~Hz}, \mathrm{POCH}_{2} \mathrm{CH}_{3}\right)$, $3.75-3.99\left(8 \mathrm{H}, \mathrm{m}, \mathrm{P}-\mathrm{OCH}_{2} \mathrm{CH}_{3}\right), 5.60(1 \mathrm{H}, \mathrm{s}, \mathrm{NH}), 5.05-$ 5.35 (1H, m, PCH), 7.89 (1H, s, Ar-H), $8.20(1 \mathrm{H}, \mathrm{s}, \mathrm{Ar}-\mathrm{H})$. ${ }^{13} \mathrm{C}-\mathrm{NMR}\left(100 \mathrm{MHz}, \mathrm{DMSO}-d_{6}\right) \delta: 15.6\left(\mathrm{~d},{ }^{3} J_{\mathrm{P}-\mathrm{C}}=5.8 \mathrm{~Hz}, \mathrm{P}-\right.$ $\left.\mathrm{OCH}_{2}-\mathrm{CH}_{3}\right), 58.5\left(\mathrm{t},{ }^{1} J_{\mathrm{PC}}=157.6 \mathrm{~Hz}, \mathrm{PCH}\right), 62.7\left(\mathrm{~d},{ }^{2} J_{\mathrm{P}-\mathrm{C}}=\right.$ $7.6 \mathrm{~Hz}, \mathrm{P}-\mathrm{OCH}_{2} \mathrm{CH}_{3}$ ), 113.9 (C-2), 115.7 (C-5), 115.8 (C-6), 118.5 (C-3), 123.1 (C-4), 140.3 (C-1). ${ }^{31} \mathrm{P}-\mathrm{NMR}(161.7 \mathrm{MHz}$, DMSO- $\left.d_{6}\right) \delta$ : 22.5. IR (KBr) $\mathrm{cm}^{-1}: 3369(\mathrm{NH}), 1240(\mathrm{P}=\mathrm{O})$, $750 \quad\left(\mathrm{P}-\mathrm{C}_{\text {aliphatic }}\right)$. ESI-MS $(\mathrm{m} / \mathrm{z}): 463 \quad\left(\mathrm{M}^{+}\right)$. Anal. Calcd for $\mathrm{C}_{15} \mathrm{H}_{25} \mathrm{Cl}_{2} \mathrm{NO}_{7} \mathrm{P}_{2}: \mathrm{C}, 38.81 ; \mathrm{H}, 5.43 ; \mathrm{N}, 3.02$. Found $\mathrm{C}$, 38.77; H, 5.38; N, 2.97.

Tetraethyl(pyridin-4-ylamino)methylenebisphosphonate (3j): White solid, yield: $90 \%$, mp $142-144^{\circ} \mathrm{C} .{ }^{1} \mathrm{H}-\mathrm{NMR}(400 \mathrm{MHz}$, DMSO- $\left.d_{6}\right) \delta: 1.10\left(6 \mathrm{H}, \mathrm{t},{ }^{3} J_{\mathrm{PH}}=8.9 \mathrm{~Hz}, \mathrm{POCH}_{2} \mathrm{CH}_{3}\right), 1.20$ $\left(6 \mathrm{H}, \mathrm{t},{ }^{3} J_{\mathrm{PH}}=9.2 \mathrm{~Hz}, \mathrm{POCH}_{2} \mathrm{CH}_{3}\right), 3.80-4.10(8 \mathrm{H}, \mathrm{m}, \mathrm{P}-$ $\left.\mathrm{OCH}_{2} \mathrm{CH}_{3}\right), 4.95-5.20(1 \mathrm{H}, \mathrm{m}, \mathrm{PCH}), 5.00(1 \mathrm{H}, \mathrm{s}, \mathrm{NH})$, $6.70(2 \mathrm{H}, \mathrm{d}, J=8.8 \mathrm{~Hz}, \mathrm{Ar}-\mathrm{H}), 8.40(1 \mathrm{H}, \mathrm{d}, J=7.6 \mathrm{~Hz}, \mathrm{Ar}-\mathrm{H})$. 
${ }^{13} \mathrm{C}-\mathrm{NMR}\left(100 \mathrm{MHz}, \mathrm{DMSO}-d_{6}\right) \quad \delta: 15.8\left(\mathrm{~d},{ }^{3} J_{\mathrm{P}-\mathrm{C}}=5.8 \mathrm{~Hz}\right.$, $\left.\mathrm{P}-\mathrm{OCH}_{2}-\mathrm{CH}_{3}\right), \quad 54.9\left(\mathrm{t},{ }^{1} J_{\mathrm{PC}}=163.4 \mathrm{~Hz}, \mathrm{PCH}\right), 63.5 \quad(\mathrm{~d}$, $\left.{ }^{2} J_{\mathrm{P}-\mathrm{C}}=7.6 \mathrm{~Hz}, \mathrm{P}-\mathrm{O} \underline{C H}_{2} \mathrm{CH}_{3}\right), 159.2$ (C-2), 107.2 (C-5), 110.3 (C-3), 150.5(C-6), 156.7 (C-4). ${ }^{31} \mathrm{P}-\mathrm{NMR}$ (161.7 MHz, DMSO$\left.d_{6}\right) \delta$ : 18.52. IR (KBr) $\mathrm{cm}^{-1}: 3252(\mathrm{NH}), 1235(\mathrm{P}=\mathrm{O}), 770$ $\left(\mathrm{P}-\mathrm{C}_{\text {aliphatic }}\right)$. ESI-MS $(\mathrm{m} / \mathrm{z})$ : $380 \quad\left(\mathrm{M}^{+}\right)$. Anal. Calcd for $\mathrm{C}_{14} \mathrm{H}_{26} \mathrm{~N}_{2} \mathrm{O}_{6} \mathrm{P}_{2}: \mathrm{C}, 44.21 ; \mathrm{H}, 6.89 ; \mathrm{N}, 7.37$. Found C, 44.17; H, $6.86 ; \mathrm{N}, 7.33$.

\section{Results and Discussion}

Synthesis The conventional method of this 3 component one pot reaction requires high temperatures and long reaction times to afford the corresponding bisphosphonates. ${ }^{20)}$ A new method is presently developed for the preparation of bisphosphonates from a mixture of amine, diethyl phosphite and triethylorthoformate in the presence of amberlyst- 15 as catalyst goes to completion at room temperature under solvent free conditions in $90 \mathrm{~min}$ and afforded high yields. Further after completion of the reaction the catalyst can be separated simply by filtration and reused for another $4-5$ times without losing its activity. ${ }^{21)}$

To optimize the reaction conditions, the reaction of 4 nitroaniline, diethyl phosphite and triethylorthoformate was selected as a model. This reaction has been performed in different organic solvents such as toluene, methanol, dioxane, tetrahydrofuran, acrylonitrile, chloroform, dichloromethane, diethyl ether in the presence of amberlyst- 15 at room temperature and low yields $(<40 \%)$ of the bisphosphonates were obtained in all these experiments. Use of higher amount of catalyst also did not lead to significant change in the reaction yields. The best result was obtained when the same reaction was done under solvent free conditions with small amount of catalyst.

Based on the optimized reaction conditions, a group of bisphosphonates were synthesized by the same reaction using different alkyl/aromatic/heterocyclic amines. In all the cases the reaction proceeded at room temperature in $90 \mathrm{~min}$ and gave good to excellent yields. In these experiments, the catalyst was isolated by filtration and could be reloaded with fresh reagents for further runs, thus, recyclization of the catalyst is possible without significant loss of activity. The products were obtained as solids and purified by column chromatography using silica gel as adsorbent and ethyl acetate-hexane $(1: 4)$ as eluent. The chemical structures of $\mathbf{3 a}-\mathbf{j}$ were confirmed by elemental analysis, IR, ${ }^{1} \mathrm{H}-,{ }^{13} \mathrm{C}-,{ }^{31} \mathrm{P}-\mathrm{NMR}$ and mass spectral data.

Biological Activity. Antimicrobial Activity The compounds $\mathbf{3 a}-\mathbf{j}$ were tested for in vitro antimicrobial activity against the Gram-positive bacteria Staphylococcus aureus (NCIM No. 5021), Bacillus subtilis (NCIM No. 2063), the Gram-negative bacteria Klebsiella pneumoniae (NCIM No. 2957), Proteus vulgaris (NCIM No. 2027) and fungi Fusarium solani (NCIM No. 1330), Curvularia lunata (NCIM No. 716) and Aspergillus niger (NCIM No. 596). The preliminary screening was carried out by agar disc-diffusion method. ${ }^{22}$ Chloramphenicol and Ketoconazole were used as control drugs. The observed data on the antimicrobial activity of the compounds and control drugs are given in Tables 1 and 2 .

Cells Bacterial strains $S$. aureus, B. subtilis, K. pneumoniae, $P$. vulgaris and fungi $F$. solani, $C$. lunata and $A$. niger were obtained from National Collection of Industrial Microor-

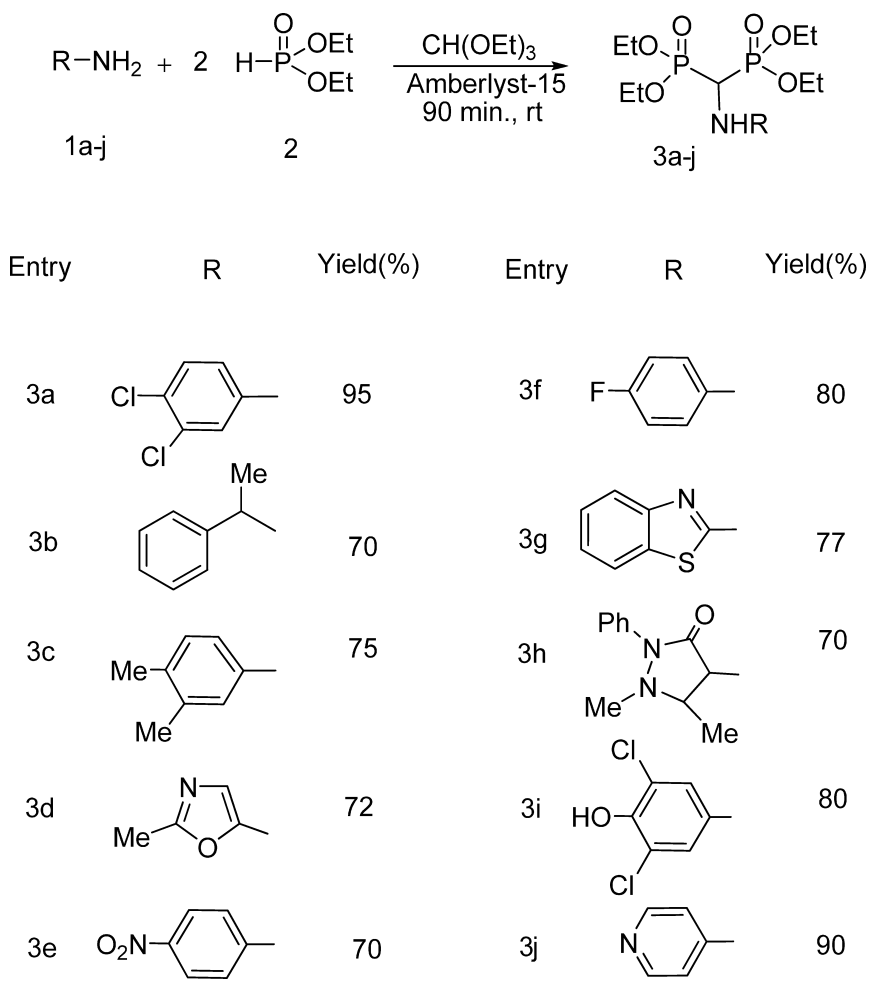

Chart 1. The Synthetic Route for Amino Methylene Bisphosphonates

ganisms (NCIM), National Chemical Laboratory, Pune, India.

Antibacterial and Antifungal Assays Preliminary antimicrobial activities of compounds $\mathbf{3 a}-\mathbf{j}$ were tested by agar disc-diffusion method. Sterile filter paper discs $(6 \mathrm{~mm}$ diameter) moistened with the test compound solution in DMSO of specific concentration $100 \mu \mathrm{M}$ and $200 \mu \mathrm{m} /$ disc were carefully placed on the agar culture plates that had been previously inoculated separately with the microorganisms. The plates were incubated at $37^{\circ} \mathrm{C}$ and the diameter of the growth inhibition zones were measured after $24 \mathrm{~h}$ in case of bacteria and after $48 \mathrm{~h}$ in case of fungi.

The results of preliminary antimicrobial testing of the compounds are shown in Tables 1 and 2. The results revealed that, both Gram-positive bacteria and Gram-negative bacteria show similar inhibitory activity. The compounds $\mathbf{3 a -}$ - and 3h showed excellent activity against Gram-positive (inhibitory zone $>25 \mathrm{~mm}$ ) and Gram-negative (inhibitory zone $>25 \mathrm{~mm}$ ) bacteria. All the test compounds showed moderate to high inhibitory effect towards tested fungi.

Antioxidant Activity The compounds $\mathbf{3 a}-\mathbf{j}$ were tested for antioxidant property by nitric oxide, ${ }^{23,24)} \mathrm{DPPH}^{25)}$ and $\mathrm{H}_{2} \mathrm{O}_{2}$ methods. ${ }^{26}$ ) The observed data on the antioxidant activity is shown in Fig. 1.

Determination of Nitric Oxide (NO) Scavenging Activity Sodium nitropruside $(5 \mu \mathrm{M})$ in phosphate buffer $\mathrm{pH} 7.4$ was incubated with $100 \mu \mathrm{M}$ concentration of test compounds dissolved in a suitable solvent (dioxane/methanol) and tubes were incubated at $25^{\circ} \mathrm{C}$ for $120 \mathrm{~min}$. Control experiment was conducted with equal amount of solvent in an identical manner. At intervals, $0.5 \mathrm{~mL}$ of incubation solution was taken and diluted with $0.5 \mathrm{~mL}$ of Griess reagent $(1 \%$ sulfanilamide, $0.1 \% \mathrm{~N}$-naphthylethylenediamine dihydrochloride and 2\%ophosphoric acid dissolved in distilled water). The absorbance 
Table 1. Antibacterial Activity of $\mathbf{3 a}-\mathbf{j}$

\begin{tabular}{|c|c|c|c|c|c|}
\hline \multirow{3}{*}{ Compound } & \multirow{3}{*}{$\begin{array}{l}\text { Concentration } \\
(\mu \mathrm{M} / \text { disc })\end{array}$} & \multicolumn{4}{|c|}{ Zone of inhibition (mm) } \\
\hline & & \multicolumn{2}{|c|}{ Gram-positive bacteria } & \multicolumn{2}{|c|}{ Gram-negative bacteria } \\
\hline & & Staphylococcus aureus & Bacillus subtilis & Klebsiella pneumoniae & Proteus vulgaris \\
\hline \multirow[t]{2}{*}{$3 \mathbf{a}$} & 100 & 29 & 28 & 30 & 32 \\
\hline & 200 & 32 & 30 & 32 & 34 \\
\hline \multirow[t]{2}{*}{$\mathbf{3 b}$} & 100 & 30 & 31 & 25 & 26 \\
\hline & 200 & 31 & 33 & 27 & 29 \\
\hline \multirow[t]{2}{*}{$3 c$} & 100 & 28 & 31 & 26 & 25 \\
\hline & 200 & 29 & 33 & 28 & 27 \\
\hline \multirow[t]{2}{*}{$3 d$} & 100 & 30 & 26 & 30 & 26 \\
\hline & 200 & 32 & 29 & 31 & 28 \\
\hline \multirow[t]{2}{*}{$3 e$} & 100 & 26 & 23 & 24 & 24 \\
\hline & 200 & 25 & 21 & 26 & 22 \\
\hline \multirow[t]{2}{*}{ 3f } & 100 & 17 & 20 & 21 & 18 \\
\hline & 200 & 19 & 21 & 22 & 19 \\
\hline \multirow[t]{2}{*}{$3 g$} & 100 & 19 & 14 & 25 & 13 \\
\hline & 200 & 18 & 15 & 26 & 14 \\
\hline \multirow[t]{2}{*}{$3 h$} & 100 & 25 & 28 & 25 & 30 \\
\hline & 200 & 27 & 30 & 26 & 31 \\
\hline \multirow[t]{2}{*}{$3 \mathbf{i}$} & 100 & 17 & 16 & 16 & 13 \\
\hline & 200 & 18 & 19 & 17 & 14 \\
\hline \multirow[t]{2}{*}{$3 \mathbf{j}$} & 100 & 14 & 18 & 19 & 17 \\
\hline & 200 & 16 & 19 & 21 & 19 \\
\hline \multirow[t]{2}{*}{ Chloramphenicol } & 100 & 35 & 38 & 37 & 42 \\
\hline & 200 & 41 & 44 & 42 & 45 \\
\hline
\end{tabular}

Table 2. Antifungal Activity of $\mathbf{3 a}-\mathbf{j}$

\begin{tabular}{|c|c|c|c|c|}
\hline \multirow{2}{*}{ Compound } & \multirow{2}{*}{ Concentration $(\mu \mathrm{M} /$ disc $)$} & \multicolumn{3}{|c|}{ Zone of inhibition (mm) } \\
\hline & & Fusarium solani & Curvularia lunata & Aspergillus niger \\
\hline \multirow[t]{2}{*}{$\mathbf{3 a}$} & 100 & 30 & 26 & 28 \\
\hline & 200 & 33 & 29 & 30 \\
\hline \multirow[t]{2}{*}{$\mathbf{3 b}$} & 100 & 31 & 29 & 27 \\
\hline & 200 & 33 & 30 & 31 \\
\hline \multirow[t]{2}{*}{$3 c$} & 100 & 27 & 29 & 28 \\
\hline & 200 & 30 & 31 & 31 \\
\hline \multirow[t]{2}{*}{$3 d$} & 100 & 28 & 27 & 27 \\
\hline & 200 & 30 & 29 & 30 \\
\hline \multirow[t]{2}{*}{$3 e$} & 100 & 23 & 22 & 26 \\
\hline & 200 & 26 & 21 & 28 \\
\hline \multirow[t]{2}{*}{$3 f$} & 100 & 16 & 18 & 17 \\
\hline & 200 & 18 & 20 & 20 \\
\hline \multirow[t]{2}{*}{$3 g$} & 100 & 19 & 19 & 18 \\
\hline & 200 & 21 & 22 & 21 \\
\hline \multirow[t]{2}{*}{$3 \mathbf{h}$} & 100 & 29 & 30 & 27 \\
\hline & 200 & 31 & 32 & 30 \\
\hline \multirow[t]{2}{*}{$3 \mathbf{i}$} & 100 & 15 & 17 & 19 \\
\hline & 200 & 17 & 18 & 20 \\
\hline \multirow[t]{2}{*}{$\mathbf{3 j}$} & 100 & 17 & 20 & 21 \\
\hline & 200 & 18 & 22 & 23 \\
\hline \multirow[t]{2}{*}{ Ketoconazole } & 100 & 38 & 41 & 36 \\
\hline & 200 & 42 & 44 & 39 \\
\hline
\end{tabular}


of the chromophore formed during diazotization of nitrite with sulfanilamide and subsequent $N$-naphthylethylenediamine dihydrochloride was read at $\lambda 546 \mathrm{~nm}$. The experiment was repeated in triplicate.

$$
\text { NO scavenged }(\%)=\frac{\left(A_{\text {cont }}-A_{\text {test }}\right)}{A_{\text {cont }}} \times 100
$$

Where $A_{\text {cont }}$ is the absorbance of the control reaction and $A_{\text {test }}$ is the absorbance in the presence of the sample.

In the case of amino methylene bisphosphonates $\mathbf{3 a}-\mathbf{j}$ derivatives 3i showed the highest $\mathrm{NO}$ scavenging with $\mathrm{IC}_{50}$ of $56.21 \mu \mathrm{M}$ when compared with other compounds. The remaining compounds exhibited reducing power activity in the following order: 3g $\left(\mathrm{IC}_{50} 58.79 \mu \mathrm{M}\right), 3 \mathbf{3 f}\left(\mathrm{IC}_{50} 63.39 \mu \mathrm{M}\right)$, 3j $\left(\mathrm{IC}_{50} 79.72 \mu \mathrm{M}\right), \mathbf{3 e}\left(\mathrm{IC}_{50} 79.81 \mu \mathrm{M}\right), 3 \mathbf{d}\left(\mathrm{IC}_{50} 86.51 \mu \mathrm{M}\right), \mathbf{3 c}$ $\left(\mathrm{IC}_{50} 86.65 \mu \mathrm{M}\right), \mathbf{3 b}\left(\mathrm{IC}_{50} 89.43 \mu \mathrm{M}\right), 3 \mathbf{h}\left(\mathrm{IC}_{50} 89.65 \mu \mathrm{M}\right)$, 3a $\left(\mathrm{IC}_{50} 90.11 \mu \mathrm{M}\right)$ and when compared with ascorbic acid $\left(\mathrm{IC}_{50}\right.$ $96.90 \mu \mathrm{M})$.

Determination of 1,1-Diphenyl-2-picrylhydrazyl (DPPH) Free Radical Scavenging Activity The nitrogen centered stable free radical 1,1-diphenyl-2-picrylhydrazyl (DPPH) has often been used to characterize antioxidants. It is reversibly reduced and the odd electron in the DPPH free radical gives a strong absorption maximum at $\lambda 517 \mathrm{~nm}$, which is purple in color. This property makes it suitable for spectrophotometric studies. A radical scavenging antioxidant reacts with DPPH stable free radical and converts into 1,1-diphenyl-2-picrylhydrazine. The resulting decolorization is stoichiometric with respect to the number of electrons captured. The change in the absorbance produced in this reaction has been used to measure antioxidant properties.

The solutions of test compounds $(100 \mu \mathrm{M})$ were added to DPPH $(100 \mu \mathrm{M})$ in dioxane/ethanol. The tubes were kept at an ambient temperature for $20 \mathrm{~min}$. and the absorbance was measured at $\lambda 517 \mathrm{~nm}$. The difference between the test and the control experiments was taken and expressed as the per cent scavenging of the DPPH radical.

In the case of amino methylene bisphosphonates $\mathbf{3 a}-\mathbf{j}$ derivatives 3i showed the highest DPPH radical scavenging activity with $\mathrm{IC}_{50}$ of $62.57 \mu \mathrm{M}$ when compared with other compounds. The remaining compounds exhibited reducing power activity in the following order: $\mathbf{3 g}\left(\mathrm{IC}_{50} 69.43 \mu \mathrm{M}\right), \mathbf{3 j}$ $\left(\mathrm{IC}_{50} 72.95 \mu \mathrm{M}\right), \mathbf{3 f}\left(\mathrm{IC}_{50} 74.57 \mu \mathrm{M}\right), \mathbf{3 e}\left(\mathrm{IC}_{50} 81.21 \mu \mathrm{M}\right), \mathbf{3 h}\left(\mathrm{IC}_{50}\right.$ $87.25 \mu \mathrm{M}), \mathbf{3 c}\left(\mathrm{IC}_{50} 87.70 \mu \mathrm{M}\right), \mathbf{3 b}\left(\mathrm{IC}_{50} 87.91 \mu \mathrm{M}\right), 3 \mathbf{a} \quad\left(\mathrm{IC}_{50}\right.$ $89.81 \mu \mathrm{M}), 3 \mathbf{d}\left(\mathrm{IC}_{50} 90.01 \mu \mathrm{M}\right)$ and when compared with ascorbic acid $\left(\mathrm{IC}_{50} 95.37 \mu \mathrm{M}\right)$.

Determination of Hydrogen Peroxide $\left(\mathrm{H}_{2} \mathrm{O}_{2}\right)$ Scavenging Activity It was determined using a modified method of Gow-Chin Yen and Hui-Yin Chen. $4 \mathrm{~mm}$ solution of $\mathrm{H}_{2} \mathrm{O}_{2}$ was prepared in phosphate-buffered saline (PBS, pH 7.4). $\mathrm{H}_{2} \mathrm{O}_{2}$ concentration was determined spectrophotometrically from absorbance at $230 \mathrm{~nm}$ using molar absorptivity $81 \mathrm{~m}^{-1} \mathrm{~cm}^{-1}$. One hundred micromolar compounds solution in $4 \mathrm{~mL}$ distilled water were added to $0.6 \mathrm{~mL} \mathrm{H}_{2} \mathrm{O}_{2}$-PBS solution. Absorbance of $\mathrm{H}_{2} \mathrm{O}_{2}$ at $230 \mathrm{~nm}$ was determined 10 min later against a blank solution containing parent compound with PBS without $\mathrm{H}_{2} \mathrm{O}_{2}$. Ascorbic acid was added in place of compound in $4 \mathrm{~mL}$ distilled water and the solution wad added to $0.6 \mathrm{~mL} \mathrm{H}_{2} \mathrm{O}_{2}$ solution in PBS. Absorbance was determined $10 \mathrm{~min}$ later against a blank solution similar to that above.

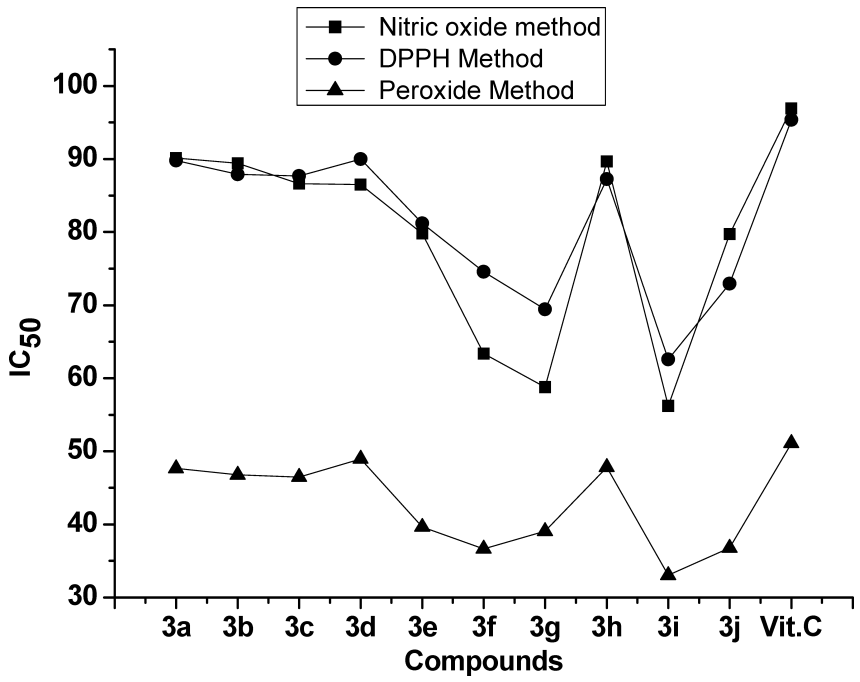

Fig. 1. Antioxidant Activity of $\mathbf{3 a -} \mathbf{j}$

In the case of amino methylene bisphosphonates $\mathbf{3 a}-\mathbf{j}$ derivatives $3 \mathbf{i}$ showed the highest $\mathrm{H}_{2} \mathrm{O}_{2}$ scavenging activity with $\mathrm{IC}_{50}$ of $33.03 \mu \mathrm{M}$ when compared with other compounds. The remaining compounds exhibited reducing power activity in the following order: 3f $\left(\mathrm{IC}_{50} 36.65 \mu \mathrm{M}\right), \mathbf{3 j}\left(\mathrm{IC}_{50} 36.80 \mu \mathrm{M}\right)$, 3g $\left(\mathrm{IC}_{50} 39.05 \mu \mathrm{M}\right), \mathbf{3 e}\left(\mathrm{IC}_{50} 39.65 \mu \mathrm{M}\right), \mathbf{3 c}\left(\mathrm{IC}_{50} 46.48 \mu \mathrm{M}\right), \mathbf{3 b}$ $\left(\mathrm{IC}_{50} 46.78 \mu \mathrm{M}\right), \mathbf{3 a}\left(\mathrm{IC}_{50} 47.65 \mu \mathrm{M}\right), 3 \mathbf{h}\left(\mathrm{IC}_{50} 47.83 \mu \mathrm{M}\right), \mathbf{3 d}$ $\left(\mathrm{IC}_{50} 48.98 \mu \mathrm{M}\right)$, and when compared with ascorbic acid $\left(\mathrm{IC}_{50}\right.$ $51.13 \mu \mathrm{M})$.

The compounds $\mathbf{3} \mathbf{a}-\mathbf{j}$ were tested for antioxidant property by Nitric Oxide, DPPH and $\mathrm{H}_{2} \mathrm{O}_{2}$ methods. The compounds 3e, 3f, 3g, 3i and 3j exhibited high antioxidant property in all the 3 methods at $100 \mu \mathrm{M}$ concentration. In fact, the compound having amino methylene bisphosphonates units displayed high antioxidant property.

Majority of the compounds showed high activities for nitric oxide, DPPH and $\mathrm{H}_{2} \mathrm{O}_{2}$ inhibition as shown in Fig. 1. The potency of these enzymes inhibition was mainly influenced by the fragments attached to the amino methylene bisphosphonates.

Compounds 3e, 3f, 3g, $\mathbf{3 i}$ and $\mathbf{3 j}$ showed appreciable antioxidant activity. Fluorine, Chlorine and $\mathrm{NO}_{2}$ substituents which attract the electron density appear to enhance antioxidant activity. Since fluorine shows highly negative inductive effect and $\mathrm{NO}_{2}$ is also highly electron withdrawing, the electron density around phosphonate moiety decreases and consequently increases affinity towards oxygen derived free radicals and mobilizes ROS to be scavenged from the living system. The presence of fluorine as a substituent also plays a determinant role in the inhibition of enzymes which has importance in drug discovery such as mimic and destabilization effects. Due to the presence of electron withdrawing groups in $\mathbf{3 a}-\mathbf{j}$, all the title compounds exhibit significant antioxidant activity.

\section{Conclusion}

An effective and simple method for the synthesis of new amino bisphosphonates $(\mathbf{3 a}-\mathbf{j})$ is reported. Compounds $\mathbf{3 a}-\mathbf{d}$ and $\mathbf{3 h}$ exhibited significant antimicrobial and $\mathbf{3 e}, \mathbf{3 f}, \mathbf{3 g}, \mathbf{3 i}$ and $\mathbf{3 j}$ exhibited significant antioxidant properties in nitric oxide, DPPH and $\mathrm{H}_{2} \mathrm{O}_{2}$ method inhibitory potency. 
Acknowledgements The authors express their grateful thanks to Prof. C. D. Reddy, Department of Chemistry, Sri Venkateswara University, Tirupati, for his helpful discussions and for BRNS, BARC, Mumbai for providing financial assistance (2010/37C/26/BRNS/1424). The authors also express their thanks to Mr. P. Ramamohan, Department of Biochemistry, S.V. University, Tirupati for conducting biologtical activity.

\section{References}

1) German J. B., Adv. Exp. Med. Biol., 459, 23-50 (1999).

2) Jacob R. A., Subcell. Biochem., 25, 1-16 (1996).

3) Knight J. A., Ann. Clin. Lab. Sci., 28, 331-346 (1998).

4) Kasser J., Nazarov A. A., Hartinger C. G., Wdziekonski B., Dani C., Kuznetsov M. L., Arion V. B., Keppler B. K., Bioorg. Med. Chem., 17, 3388-3393 (2009).

5) Russell G. G., Rogers M. J., Bone, 25, 97-106 (1999).

6) Veera Narayana Reddy M., Kishore Kumar Reddy K. R., Bhupendra Reddy C., Siva Kumar B., Suresh Reddy C., Devendranath Reddy C., J. Chem. Res., 4, 258-260 (2009).

7) Hudock M. P., Sanz-Rodríguez C. E. Y., Song Y., Chan J. M., Zhang Y., Odeh S., Kosztowski T., Leon-Rossell A., Concepción J, L., Yardley V., Croft S. L., Urbina J. A., Oldfield E., J. Med. Chem., 49, 215-223 (2006).

8) Kiran Y. B., Devendranath Reddy C., Gunasekar D., Suresh Reddy C., Leon A., Barbosa L. C. A., Eur. J. Med. Chem., 43, 885-892 (2008).

9) Green J. R., Cancer, 97 (Suppl.), 840-847 (2003).

10) Clézardin P., Ebetino F. H., Fournier P. G. J., Cancer Res., 65, 4971-4974 (2005).

11) Dunn C. J., Galinet L. A., Wu H., Nugent R. A., Schlachter S. T.,
Staite N. D., Aspar D. G., Elliott G. A., Essani N. A., Rohloff N. A., J. Pharmacol. Exp. Ther., 266, 1691-1698 (1993).

12) Flora L., Smith R. J., Arthritis Rheum., 22, 340-346 (1979).

13) Van Offel J. F., Schuerwegh A. J., Bridts C. H., Bracke P. G., Stevens W. J., De Clerck L. S., Clin. Exp. Rheumatol., 19, 13-20 (2001).

14) Singh U. S., Shankar R., Kumar A., Trivedi R., Chattopadhyay N., Shakya N., Palne S., Gupta S., Hajela K., Bioorg. Med. Chem., 16, 8482 -8491 (2008).

15) Dombrecht E. J., Cos P., Vanden Berghe D., Van Offel J. F., Schuerwegh A. J., Bridts C. H., Stevens W. J., De Clerck L. S., Biochem. Biophys. Res. Commun., 314, 675-680 (2004).

16) Cantatore F. P., Acquista C. A., Pipitone V., J. Rheumatol., 26, 2318-2323 (1999).

17) Balakrishna A., Narayana Reddy M. V., Rao P. V., Kumar M. A., Kumar B. S., Nayak S. K., Reddy C. S., Eur. J. Med. Chem., 46, 1798-1802 (2011).

18) Ko S., Yao C. F., Tetrahedron Lett., 47, 8827-8829 (2006).

19) Liu Y. H., Liu Q. S., Zhang H., J. Mol. Catal., 296, 42-46 (2008).

20) Takeuchi M., Sakamoto S., Yoshida M., Abe T., Isomura Y., Chem. Pharm. Bull., 41, 688-693 (1993).

21) Tajbakhsh Md., Heydari A., Khalilzadeh Md. A., Synlett, 15, 2347-2350 (2007)

22) Mangte D. V., Deshmukh S. P., Bhokare D. D., Deshponde Arti R., Indian J. Pharm., 69, 295-298 (2007).

23) Shirwaiker A., Rajendran K., Dinesh Kumar C., Indian J. Exp. Biol., 42, 803-807 (2004).

24) Babu B. H., Shailesh B. S., Paddikala J., Fitotherapia, 72, 272-277 (2001).

25) Kato K., Terao S., Shimamoto N., Hirata M., J. Med. Chem., 31, 793-798 (1988).

26) Gow-Chin Y., Hui-Yin C., J. Agric. Food Chem., 43, 27-32 (1995). 